Fabian Grumbrecht

\title{
"What are you doing, Dave?": The Confrontation of Dave Bowman and HAL 9000 in Stanley Kubrick's 2001: A Space Odyssey
}

\section{Introduction}

The genre of Science Fiction film has been frequently marked by heated battles between human beings and various menacing forces. These forces are mainly embodied by alien races (Invasion of the Body Snatchers [1956], Alien [1979]), mutated life forms (Tarantula [1955]), dystopian regimes (Brazil [1985], Nineteen Eighty-Four [1984]), or rebellious artificial intelligences. Within the last-mentioned category, one example of a computer turning against human beings can be found in Stanley Kubrick's 2001: A Space Odyssey (1968), namely: HAL 9000. In addition to the computer's menacing act of murdering four astronauts, scholars and critics like Michael Mateas have focused on HAL's apparent "emotional and psychological depth" (106). The computer appears in the film's second part "JUPITER MISSION, 18 MONTHS LATER." Barton Palmer has pointed out that this part "is handled in more or less traditional Hollywood fashion" (19). Moreover, Mateas adds that HAL primarily serves "a function within the plot" (123) until he is deactivated by the astronaut Dave Bowman. HAL's unexpected and enigmatic malfunction turns the computer into the antagonist of the film when it attempts to 
kill the crew of the spaceship Discovery. Consequently, Dave Bowman, as the only surviving crewmember, is generally identified as HAL's counterpart. This constellation culminates in a confrontation, in which the astronaut wants to turn the computer off.

In this chapter I would like to analyze the confrontation of Dave Bowman and HAL, because it may not necessarily be classified as a "big battle" (Mamber 64). Instead, the showdown between HAL and the astronaut is not marked by physical violence. It is rather portrayed in a unique way as an almost trivial act of shutting down a technical apparatus, an act that is completely devoid of violent struggles. Nevertheless, the confrontation is subtly enhanced on the visual and aural levels. Consequently, this article will focus on HAL's status as an antagonist to Dave Bowman, followed by the analysis of the visual and aural presentation of the confrontation and interaction of the two characters on the level of plot.

\section{HAL's Status as an Antagonist}

HAL's antagonistic status can be inferred from Dave Bowman's position as a protagonist, which is established in the course of the plot and emphasized during the confrontation. At first, Dave Bowman is introduced as the equal of his fellow astronaut Frank Poole (Booker 86), but when Dave becomes the only surviving member of the crew on board of the Discovery, the focus on him as the protagonist increases in a significant way. Furthermore, Dave is the only character who arrives at Jupiter - the place the human characters want to reach -, and in the end becomes the Star Child. These aspects do not only single him out from his fellow astronauts but also from the scientist squad of Dr. Heywood Floyd, because their progress is either thwarted (in the case of the other astronauts) or presented en passant (in the case of Dr. Floyd, who discovers the monolith located on the moon but is not part of the monumental expedition to Jupiter).

While the plot of the whole film seems to underline Dave Bowman's position as a protagonist, the "revolt" (Booker 87) of HAL resembles an obstacle to the astronaut's progress: According to Philip Kuberski, the effect of "HAL's cold violence" (69) on Dave "prepares him for the psychic journey" (69) after the computer's demise. Therefore, HAL is indirectly marked as an antagonist and, according to Mateas, "functions as a character within the narrative machinery" (116) by counteracting Dave.

\section{The Visual Level}

The actual showdown between the astronaut and the computer sets in after Dave has managed to leave his space pod and returns to the Discovery. Meanwhile HAL has succeeded in killing the other astronauts, but its strategy of murdering Dave 
has failed. While the computer has ambushed and directly attacked the rest of the crew (the scientists in their sleep and Poole by a separation of his air hose in outer space), Dave has enough time to intrude into the spaceship after HAL has denied him access. The fact that Dave survives underlines his pivotal status in the plot and leads to the actual confrontation between him and the antagonistic computer.

While the astronaut is on his way to HAL's core the low angle of the camera dominates the scene. Mostly over-the-shoulder shots and medium shots are given by what Kevin L. Stoehr describes as "a subjective use of handheld camera ... that reveals Bowman's unique personal perspective and emotional context" (124). Then Dave climbs up the ladder, and when he walks through the crammed spaces, he seems to tower over the audience. Hence, the camera does not only mediate Dave's point of view but also assumes an external perspective. This perspective is influenced by the narrow architecture of the Discovery and makes the protagonist appear in an almost imposing way until he arrives at the small door which leads to the computer's brain room.

The first shot inside of the computer's core is taken from the actual point of view of the virtually omnipresent and "bodiless HAL" (Mateas 111). Situated in what Stephen Mamber describes as the "geometrical space ... of the memory core" (64), the technical devices and modules reveal the computer's 'physical' location. By entering this room, the protagonist is able to participate in a genuine 'confrontation' with an artificial intelligence that seems to be impalpable.

In contrast to the previous sequences of this section, the iconic shot of "HAL's red-lit eyeball" (Stoehr 127) is not depicted as extensively as before. Earlier in the film, HAL's red eye functioned as the main visualization of the artificial intelligence as a character. It was furthermore accompanied by the use of a fisheye lens that emphasized the look through HAL's eye. The computer's "subjective view" (Mateas 108) is completely abandoned during the showdown. By neglecting the tendency to "use his vision in pursuit of goals" (Mateas 108), HAL seems to focus on the use of its voice instead. ${ }^{1}$ Full shots of Dave's body in the core alternate with shots of his body reflection in the artificial eye and serve thus to portray the two as opponents.

According to J. P. Telotte, the protagonist "moves at odd angles and even floats ... as he deactivates HAL" (49). This is especially evident when Dave Bowman approaches the computer's modules: Suddenly, the camera's position changes twice, thus evoking the illusion that Dave does no longer float in a horizontal position while facing the room's ceiling. After a sudden cut, the camera's position has turned upside down and after a second cut, a medium shot portrays Dave sideways. He seems to stand vertically in front of the modules now. This change in position seems to establish a more matter-of-fact portrayal of the astronaut's following actions, which rather resemble a customary behavior instead of a

HAL's voice is analyzed in section four of this article. 
confrontation with an antagonist in a state of weightlessness. An over-theshoulder shot reveals the various modules Dave works on in order to shut HAL down. During this process, a close-up of Dave's head gives way to his neutral facial expressions while the technical devices are mirrored in the visor of his helmet, resembling the apex of the way the two characters "are superimposed several times" (Mamber 64).

On the whole, the depictions of Dave seem to prevail on the visual level. But how could the widespread interpretation of HAL's "establishing empathy with the audience" (Mateas 116) have been accepted and emphasized, when the computer's visual point of view (as an obvious device for creating identification with a character in a film) is neglected in this sequence? The reasons for this interpretation may be found on the aural level.

\section{The Level of Sound}

There is no underlying soundtrack; instead the constant noise of the air supply and breathing (introduced during Poole's examination of "the ship's AE-35 unit" [Booker 78]) dominate this section of the film. These sounds replace dramatizing music which is mostly used in films of various genres in order to underline the final confrontation of protagonist and antagonist. The "vivid contrast between sound and silence" (Landy 96) used during the death of Poole and the other astronauts is no longer employed. In addition to his own breathing, Dave and the audience also perceive HAL's "range of natural language competencies" (Mateas 107), performed by Douglas Rain.

Once the computer's iconic visual representation (i.e. the red eye) is no longer predominating, HAL is mainly embodied by its voice. HAL tries to apologize for its actions and to reason with Dave, for example by saying, "I know I've made some very poor decisions recently." Two interpretations regarding the effect of the computer's utterances may be contrasted: The idea that the computer's demise evokes "sadness and pity" (Mateas 116) in the audience as part of an "ambiguous double reading" (Mateas 110), and the view that "HAL's actions mime human limitations in thinking and decision making" (Landy 96). It can be argued that the aural level contradicts the visual level when Dave enters the brain room. HAL's voice and remarks are used to conceal what the sight of the modules and technical devices in its core affirms. HAL may be able to use "general language and reasoning capabilities" (Mateas 107) and he seems to voice "inner conflicts" (107), yet the pleading, "disembodied voice" (Landy 96) contrasts with the artificial structure of the computer's electronic nerve centre. As a result, it becomes obvious that HAL is a highly developed computer instead of a living creature. In his role as antagonist, HAL uses its voice as a tool of deception and manipulation, but this strategy is exposed when Dave confronts the actual origin of the voice (i.e. the artificial structure of the modules). 
Because of the combination of sounds (especially the stream of oxygen, the breathing, and HAL's voice), Dave's point of view dominates on the aural level. All the sounds he perceives in his suit are transmitted and the absence of a soundtrack or sound effects contributes to a sober and plain presentation, which is devoid of commentative sounds that are usually employed in films in order to increase tension. Dramatizing aural effects are not used in an excessive manner; even Dave's way of breathing seems to be controlled: He does not hyperventilate; instead his breathing is determined by his physical actions (e.g. climbing up the ladder, walking through the corridors, floating through the brain room).

\section{The Interaction of HAL and Dave}

The final and confrontational encounter of protagonist and antagonist is marked by a number of peculiarities. HAL tries to counteract and stop Dave Bowman by corresponding to his emotions instead of using brute violence, although the computer killed Frank Poole and the three sleeping crewmen. According to Telotte, it seems as if HAL virtually "controls all functions, including life support on board Discovery" (48). Yet after Dave has managed to enter the ship again, the computer resumes a non-violent strategy.

Therefore, HAL's control over the ship does not turn into a life-threatening danger for Dave. Although the human characters are "dependent for their life functions on" (Landy 97) the computer, they have to assume a special state before they fall victim to his "goal-driven behavior" (Mateas 108): The three hibernating scientists are killed while they are defenceless and Poole has to exit the Discovery in his space suit before HAL attacks him by seizing control over his space pod. Moreover, the computer's attempt at eliminating Dave also involves excluding him from the spaceship once HAL sees that Bowman has forgotten his helmet. Yet the protagonist's return to the spaceship does not trigger a similar attempt targeted at HAL. Although HAL's "function is to manage the ship" as Mateas puts it (107), he either has to make use of his victims' defenceless and inactive state (in the case of the sleeping astronauts), or he has to lure them into space (in the case of Frank Poole and Dave Bowman) instead of violently confronting them on board of the Discovery. Having overcome the computer's insidiousness, Dave's special status as the protagonist of the film is implicitly underlined while HAL changes its strategy.

Dave does not react to HAL's voice (until he answers the antagonist's offer to sing a song). Instead, the protagonist proceeds to shut down the computer in a matter-of-fact way, acting extremely unfazed and seemingly without showing any emotions. While this behavior of Dave Bowman (and also Frank Poole) let HAL appear "more human and empathetic" (Stoehr 125) in the previous sequences, now it makes the portrayal of the confrontation appear almost trivial. It may be doubted that Palmer's definition of the confrontation as a "struggle, which is 
more physical than psychological and climaxes in ... violence" (19) is appropriate. Landy points out that no "vivid and excessive images of mutilation" (97) are employed: HAL is not destroyed in the course of a heated battle, but turned off very soberly. Likewise, the astronaut has to protect himself mentally from succumbing to HAL's simulated reasoning and emotions (e.g. "I'm afraid, Dave") and is not confronted with some kind of a violent assault unleashed by the computer. What some critics and scholars who tend to humanize the antagonist describe as an act of "lobotomizing HAL's computer brain" (Landy 96) is actually the deactivation of "HAL's higher mental functions" (Mateas 116), while HAL's lack of another opportunity to kill Bowman makes him use his voice as a device of selfprotection.

Although Landy argues that "Dave's action suggests that his motives are tinged with vengefulness" (97), he neither smashes the artificial eye or modules, nor does he tear them out in a fit of rage. Some pragmatic reasons may be given for this behaviour. Managing to ignore the computer's voice, the protagonist is not able to deactivate HAL in frenzy or to completely destroy the computer without risking his own life (or the success of the mission). In this respect, "the film's tampering with conventional cinematic language" (97) as pointed out by Landy is determined by the circumstances given in the fictional world, because the computer's "primary function is to manage the ship and participate in the mission" (Mateas 107). Consequently, Dave's very calm reaction to HAL's live-threatening behavior does not only remain peculiar on the visual and aural level, but is also motivated on the level of plot. Although all physical actions in the final confrontation are enacted by the protagonist, HAL is actually not "heroically confronted and destroyed" (Kuberski 70) in a battle but simply shut down. What Landy describes as Dave's apparent "precipitousness" (97) only seems to make the interaction of the two characters appear one-sided because of the computer's apologetic and reasoning verbal strategy, which, however, only enhances the peculiarity on the level of sound.

Only when Dave Bowman begins to shut down HAL's memory circuit, one can assume that the protagonist has won the battle against the computer. Booker interprets the way HAL's voice slows down while singing "Daisy" as "a reversion to "childhood" (86). What Booker calls an "almost trance-like" (86) behavior as a human character on board of the Discovery has allowed Dave to overcome his opponent. Finally, the astronaut answers HAL when the computer's demise is inevitable and his utterances may not be targeted at deceiving Dave anymore. It is striking that HAL's use of its voice in order to prevent the "dismantling" (Palmer 22) does not succeed on the level of plot but influences the audience's empathy. Strictly speaking, the spectators are likely to be deluded by HAL, while Bowman manages to remain unaffected. This is achieved by contrived aspects such as the computer's age (Booker 86-87), or the way HAL sings "Daisy" while slowing down, but without making use of melodramatic elements. Only at this point 
(when the antagonist's decline has already set in), the spectators' possible "sadness and pity" (Mateas 116) may be partly justifiable. Yet, Hal's status as the film's antagonist, as well as what Landy calls "malevolent instigator of violence" (96), should not be completely overshadowed by this fairly one-sided and emotional reading.

\section{Conclusion}

In the course of 2001: A Space Odyssey, there is no heated battle between the protagonist and the villain. HAL is not destroyed, but turned off in a rather sober and trivial way. The unemotional portrayal of the showdown is achieved by the interaction of the visual and aural levels which both correspond and contrast. The visualization of the sequence is dominated by the camera focus on Dave Bowman's actions (e.g. by the use of over-the-shoulder shots and changing camera positions in between the shots) instead of assuming the computer's point of view. On the level of sound, the astronaut's perspective is underlined by the transmission of all aspects influencing his aural perception in the space suit. Furthermore, HAL attempts to exert psychological influence on the protagonist. The computer is unable to murder the astronaut because Dave is not in a state of hibernation and because he has re-entered the spaceship Discovery. Faced with the fact that Dave is on his way to HAL's core, the computer tries to prevent its disconnection by uttering apologies and remorse. Likewise, the conditions on board of the spaceship require that Dave faces the antagonist in a non-violent and calm way.

On the surface, a simple and obvious contrast between the almost "robotic" (Booker 86) actions of the astronaut and a computer who "seems more human and empathetic" (Stoehr 125) is established. However, the portrayal of HAL's core reveals that the computer' emotions, which fail to deceive Dave Bowman, are actually simulated. As a result, a certain ambiguity and a hint at banality are established by the cinematography of the showdown: The fact that the computer is turned off in a calm and matter-of-fact manner evokes trivial overtones. Dave's portrayal within the core thus makes him resemble a computer operator rather than an astronaut fighting for his life against a murderous artificial intelligence.

The two characters are confronted in the only section of the film that makes use of a conventional Hollywood-type plot (Palmer 19). However, the realization of the actual showdown does not come across as bombastic or dramatic. Consequently, the contrast between the plot and the way the showdown is displayed is highlighted even more, because the trivial and non-violent approach is usually not part of traditional Hollywood movies which mainly rely on physical clashes and vivid destruction.

The non-violent confrontation can be seen as an innovation and it has remained exceptional in the genre of Science Fiction film to this date. Hence, the tactics and demises of other vile artificial intelligences in motion pictures of the 
Fabian Grumbrecht

following decades: Proteus IV in Demon Seed (1977) turns a house into a dangerous environment in order to trap and impregnate his inventor's wife. Similar to HAL, this villainous computer is also embodied by a strikingly memorable voice (provided by Robert Vaughn). At the same time, though, it contrasts with the antagonist of 2001: A Space Odyssey by making brutal use of lasers and arm-like devices. Moreover, the Ultimate Computer in Superman III (1983) does not voice its concerns, but turns a human being into an android and emits rays in order to prevent the other characters from turning it off. As another very famous example of an antagonistic computer system, Skynet in The Terminator (1984) makes use of androids and robots in order to attack mankind and, as the main kernel of the film's plot, sends them back in time in order to indemnify its own existence and to endanger the human characters. All of those antagonistic computers make use of violence and also the protagonists in the respective films tend to defend themselves by sheer force. While HAL's defeat has sometimes been parodied and mocked in episodes of American TV-series like Parker Lewis Can't Lose (1991) or The Simpsons (2001), the final interaction between HAL and Dave has not become a paragon for showdowns between humans and computers moving beyond conventional (and primarily physical) clashes. As a result, the filmic realization of the showdown is exemplary for the whole film in having "no peers or direct successors" (Booker 88).

\section{Films Cited}

2001: A Space Odyssey. Dir. Stanley Kubrick. Perf. Keir Dullea, Gary Lockwood, William Sylvester. MGM, 1968. DVD.

Demon Seed. Dir. Donald Cammell. Perf. Julie Christie, Fritz Weaver, Gerrit Graham. MGM, 1977. DVD.

Superman III. Dir. Richard Lester. Perf. Christopher Reeve, Richard Pryor, Jackie Cooper. Warner Bros. Pictures, 1983. DVD.

The Terminator. Dir. James Cameron. Perf. Arnold Schwarzenegger, Michael Biehn, Linda Hamilton. Orion Pictures, 1984. DVD.

\section{Works Cited}

Booker, Marvin Keith. "2001: A Space Odyssey." Alternate Americas: Science Fiction Film and American Culture. Westport, CO: Praeger, 2006. 75-89. Print.

Kuberski, Philip. “Kubrick's Odyssey: Myth, Technology, Gnosis.” Arizona Quarterly: A Journal of American Literature, Culture, and Theory 64.3 (2008 Autumn): 51-73. Print. 
Landy, Marcia. "The Cinematographic Brain in 2001: A Space Odyssey.” Stanley Kubrick's 2001: A Space Odyssey: New Essays. Ed. Robert Kolker. New York: Oxford UP, 2006. 87-104. Print.

Mamber, Stephen. “Kubrick in Space.” Stanley Kubrick's 2001: A Space Odyssey: New Essays. Ed. Robert Kolker. New York: Oxford UP, 2006. 55-68. Print.

Mateas, Michael. "Reading HAL." Stanley Kubrick's 2001: A Space Odyssey: New Essays. Ed. Robert Kolker. New York: Oxford UP, 2006. 105-25. Print.

Palmer, Barton. “2001: The Critical Reception and the Generation Gap.” Stanley Kubrick's 2001: A Space Odyssey: New Essays. Ed. Robert Kolker. New York: Oxford UP, 2006. 13-27. Print.

Stoehr, Kevin L. "2001: A Philosophical Odyssey." The Philosophy of Science Fiction Film. Ed. Stephen M. Sanders. Lexington, KY: U of Kentucky P, 2008. 119-33. Print.

Telotte, J. P. “The Gravity of 2001: A Space Odyssey." Stanley Kubrick's 2001: A Space Odyssey: New Essays. Ed. Robert Kolker. New York: Oxford UP, 2006. 43-53. Print. 